\title{
Effect of the diaphragmatic contraction on lower oesophageal sphincter pressure in man
}

\author{
R K MITTAL, D F ROCHESTER, AND R W MCCALLUM \\ From the Department of Internal Medicine, University of Virginia School of Medicine, Charlottesville, \\ $V A, U S A$
}

SUMMARY The effect of diaphragmatic contraction and relaxation on the lower oesophageal sphincter (LOS) pressure was studied in 10 healthy volunteers. Pressures in the oesophagus, LOS, and stomach were measured in three phases. Phase I, end tidal expiration; phase II, subjects inspired to total lung capacity (TLC) and kept the airway open (sustained diaphragmatic contraction); and phase III, at TLC subjects relaxed against a closed glottis (diaphragm relaxed). The LOS pressure in phase II was significantly higher than in phase I, while in phase III it was significantly lower compared with phase I. There was a net increase in LOS pressure of $32 \mathrm{mmHg}$ in phase II as compared to phase I. After phase II, as subjects moved into phase III, there was a sudden drop in the LOS pressure. The LOS pressure in phase II correlated with the transdiaphragmatic pressure in a linear fashion. These observations suggest that diaphragmatic contraction in man enhances the LOS pressure and this augmentation of sphincter tone may be a vital component of the antireflux mechanism.

The tonic activity of the smooth muscle of the lower oesophageal sphincter (LOS) generates resting lower oesophageal sphincter pressure' and this sphincteric mechanism is considered to be the major antireflux barrier. Interestingly, the sling fibres of the right crura of the diaphragm are arranged in such a way that they could also exert a sphincteric action at the lower oesophageal sphincter. ${ }^{2}$ Recent studies suggest that the respiration induced pressure oscillations observed in the cat LOS during manometric recordings are the result of an active diaphragmatic contraction. ${ }^{3}$ The studies by Dodds et al suggested that these pressure oscillations are caused by a relative movement of the LOS over a fixed manometric recording site. ${ }^{+}$Whether the diaphragmatic contraction could actually enhance the LOS pressure in man is not yet clear. The purposes of our study were to: (1) examine the effects of diaphragmatic contraction and relaxation on the LOS pressure in healthy volunteers, and (2) determine if the increase in gastroesophageal gradient associated with diaphragmatic contraction is associated with acid reflux into the oesophagus in normal subjects.

Address for correspondence: Ravinder K Mittal. MD. Box 145. Department of Internal Medicine. University of Virginia Medical Center, Charlottesville, VA 22908, USA.

Received for publication 8 May. 1987.

\section{Methods}

SUBJECTS

Oesophageal manometric and $\mathrm{pH}$ studies were carried out in 10 healthy volunteers. The group was comprised of six men and four women with a mean age of 25.7 years (range 19-32). All the subjects denied a history of heartburn or any symptom suggestive of oesophageal disease. None were taking any medication. The protocol for the study was approved by the Human Investigation Committee at the University of Virginia.

After a six to eight hour fast, a manometric catheter with a $6 \mathrm{~cm}$ sleeve device (Arndorfer Medical Specialty Inc, Milwaukee, WI) was placed through the nose into the oesophagus and stomach. In five subjects an intraluminal micro $\mathrm{pH}$ electrode was attached to the manometric catheter so as to record intraoesophageal $\mathrm{pH} 5 \mathrm{~cm}$ above the LOS. The sleeve device and the side hole above the sleeve device were first positioned into the stomach. The manometric assembly was then withdrawn in a stepwise fashion until the side hole at the proximal end of the sleeve recorded intraoesophageal pressure just above the LOS and the proximal end of the sleeve straddled the LOS. The gastric pressure was measured from a side hole located $2 \mathrm{~cm}$ distal to the 
lower end of the sleeve. Intraoesophageal pressure was also monitored at 5 and $10 \mathrm{~cm}$ above the LOS. Ten, $5 \mathrm{ml}$ water swallows were performed in each subject to confirm the normal oesophageal motility.

Pressures in the oesophagus, LOS and stomach were then monitored during three phases with the subjects lying supine on a stretcher. Phase I represented end tidal expiraton. In phase II, subjects were asked to inspire to total lung capacity and then maintain the airway open so that they had to sustain diaphragmatic contraction. Evidence of the sustained effort in this phase was provided by the sustained negative and positive pressures in the oesophagus and stomach respectively. Subjects remained in this phase until the LOS recording showed a stable and horizontal pattern. In phase III, while the subjects were still at total lung capacity they were instructed to relax against the closed glottis. This manoeuvre causes relaxation of the diaphragm. ${ }^{56}$ Equalisation of the oesophageal and gastric pressures confirmed that the subjects had actually moved into phase III. Subjects remained in phase III for 10-15 seconds and then expired air out of their lungs.

The response rate of the sleeve device is dependent upon the actual position of the LOS along the length of the sleeve device.' We reasoned that in phase II when the subject inspires to total lung capacity the
LOS would move from proximal to the distal end of the sleeve, because the LOS moves passively with the diaphragm whereas the manometric catheter remains stationary. ${ }^{x "}$ Therefore, it was vital to measure the response rate of the sleeve at its proximal and distal ends so as to determine the time that the subjects would be required to sustain diaphragmatic contraction in phase II that would permit an accurate measurement of the LOS pressure. An in vitro study with the sleeve device revealed that at a rate of perfusion of $0.6 \mathrm{ml} / \mathrm{min}$, a sudden occlusion of the sleeve at its proximal end results in a rise in pressure of $150 \mathrm{mmHg}$ in less than one second. On the other hand, a sustained occlusion of 17 seconds was required to generate a pressure of $150 \mathrm{mmHg}$ at the distal end of the sleeve. The drop in pressure after occlusion is instantaneous at proximal as well as at the distal end of the sleeve device. Based on the in vitro study, the protocol for phase II required subjects to sustain the inspiratory effort for 17 seconds or until the LOS recording showed a horizontal pattern.

Phase I of the experiment was started at least 60) seconds after a swallow so that the LOS pressure was stable. Subjects were instructed not to swallow during any of the three phases. If a swallow or an oesophageal contraction occurred during any of

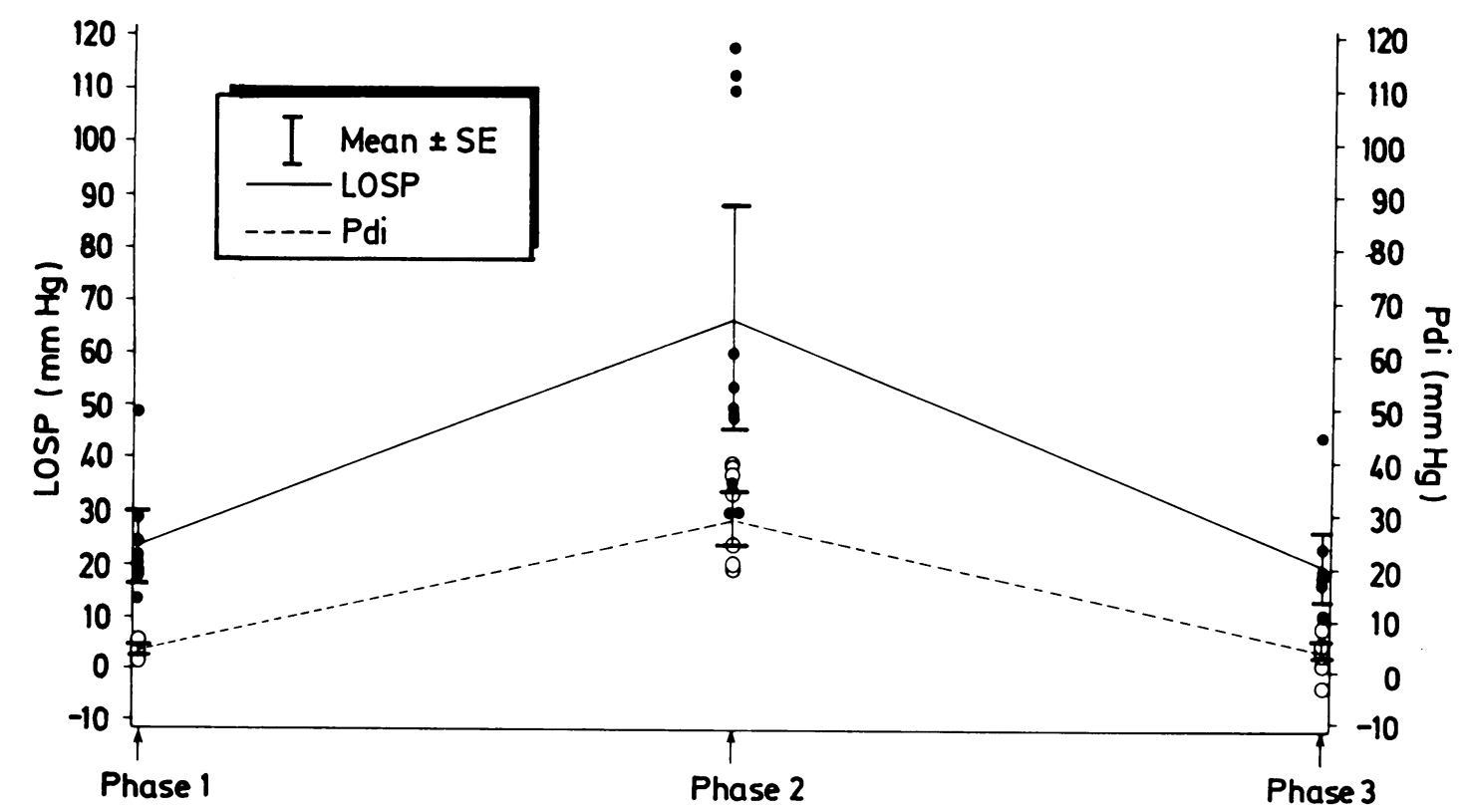

Fig. 1 Lower oesophageal sphincter pressure (LOSP) in three phases. Data are presented as mean \pm SEM. Phase I: end tidal expiration. Phase II: inspiration to total lung capacity with airway open (diaphragmatic contraction). Phase III: total lung capacity with airway closed (diaphragm relaxed). LOSP in phase II is significantly higher than phase I whereas in phase III it is significantly lower than phase 1 . 
these phases, the entire sequence was repeated. The entire sequence of phases I, II, and III was repeated successfully five times in each subject.

\section{MEASUREMENT OF PRESSURES}

The LOS and oesophageal pressures were measured in reference to the phase I end expiratory gastric pressure which was set at zero. As the gastric pressure was higher in phases II and III as compared to phase I the difference between the LOS and gastric pressure in phases II and III was called the net LOS pressure. Transdiaphragmatic pressure (Pdi) in each phase was obtained by subtracting oesophageal from the gastric pressure. Because the oesophageal pressure was always negative in reference to the gastric pressure, the Pdi was always a positive number.

\section{ACID REFLUX}

Initially an acid $\mathrm{pH}(<2 \cdot 0)$ was documented in the stomach in each subject before the probe was positioned in the oesophagus. Acid reflux from the stomach to the oesophagus was considered to have occurred when intraoesophageal $\mathrm{pH}$ fell to less than $4 \cdot 0$ for longer than five seconds.

\section{STATISTICAL ANALYSIS}

Data were analysed using signed rank tests, Spearman rank order correlation, and simple linear regression. All data are presented as mean \pm SEM.

\section{Results}

The LOS pressure in phase I was $25 \cdot 3 \pm 3 \cdot 1 \mathrm{mmHg}$. In phase II, it increased to $66 \cdot 8 \pm 10.5 \mathrm{mmHg}$ (Fig. 1). This difference is highly significant $(p<0 \cdot 005)$. The net LOS pressure - that is, LOS pressure corrected for gastric pressure - was $31.9 \pm 7.8 \mathrm{mmHg}$ higher in phase II than in phase I. The rise in LOS pressure in phase II was slow (Fig. 2) and actually in some subjects the LOS recording showed an initial drop in the LOS pressure but as the subjects remained in phase II the LOS pressure gradually increased until it reached a maximal and sustained value at which point a horizontal LOS tracing was obtained.

As the subjects moved from phase II to phase III, there was a sudden drop of LOS pressure (Fig. 2). This drop in the LOS pressure was usually greater than the initial rise of the LOS pressure that occurred from phases I to II. The LOS pressure in phase III was $3.2 \mathrm{mmHg}$ lower than phase I, a small but nevertheless statistically significant difference $(\mathrm{p}<0 \cdot 04)$.

The transdiaphragmatic pressure (Pdi) was higher in phase II than in phase I $(29 \cdot 7 \pm 2.6 v 3 \cdot 8 \pm 0 \cdot 4)$. The oesophageal and gastric pressures were nearly equal in phase III, and the Pdi was $4.3 \pm 1.0 \mathrm{mmHg}$. The

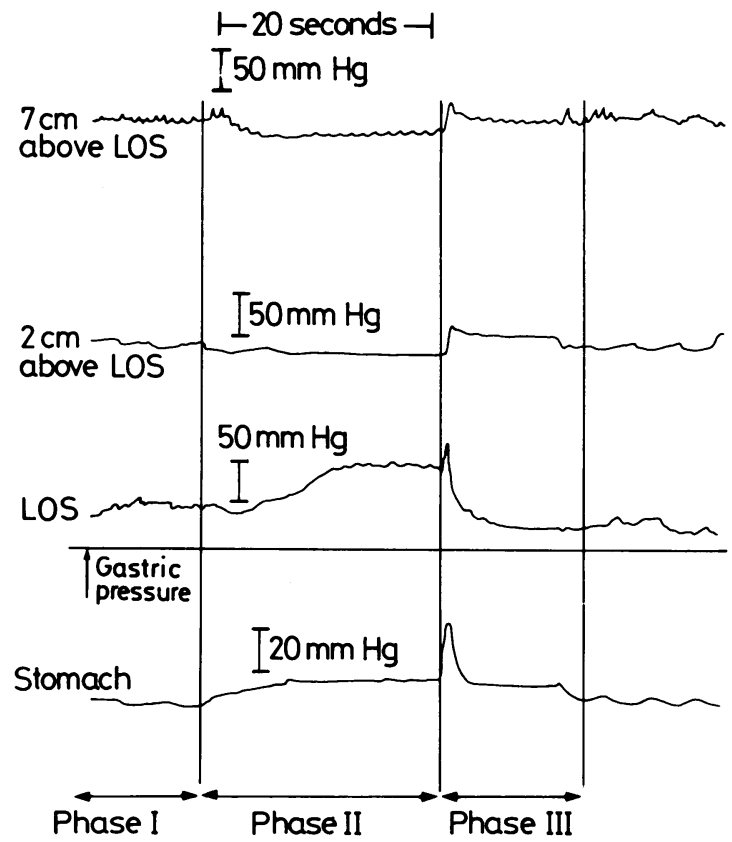

Fig. 2 Actual manometric tracing of oesophageal, lower oesophageal sphincter (LOS), and gastric pressures during phases I, II, and III in a normal subject. LOS pressure in phase II increased gradually until a horizontal pattern was attained. As subjects moved into phase III, there is an instantaneous fall in LOS pressure. LOS pressure in phase III is significantly lower than in phase I. Actual reason for transient increase in gastric pressure at the onset of phase III in this subject is not clear but most likely represents a transient Valsalva manoeuvre.

LOS pressure in phase II correlated with the Pdi in a linear fashion, $r=0.79, p<0 \cdot 005$ (Fig. 3). The net increase in LOS pressure in phase II also correlated with Pdi in a linear fashion, $r=0.77(p<0 \cdot 008)$.

In spite of a large gastro-oesophageal gradient during phase II, acid reflux did not occur in any subject. There were no episodes of acid reflux during any of these phases in any of the subjects.

\section{Discussion}

Our study shows that a physiologic manoeuvre that causes diaphragmatic contraction also results in a significant increase of the LOS pressure. Diaphragmatic relaxation, after its contraction, results in an instantaneous fall of the LOS pressure. Our study suggests that this augmentation of the LOS pressure by diaphragmatic contraction may be critical in prevention of gastro-oesophageal reflux at a time when the gastro-oesophageal pressure gradient is high.

The pinch cock action of the diaphragm can be 


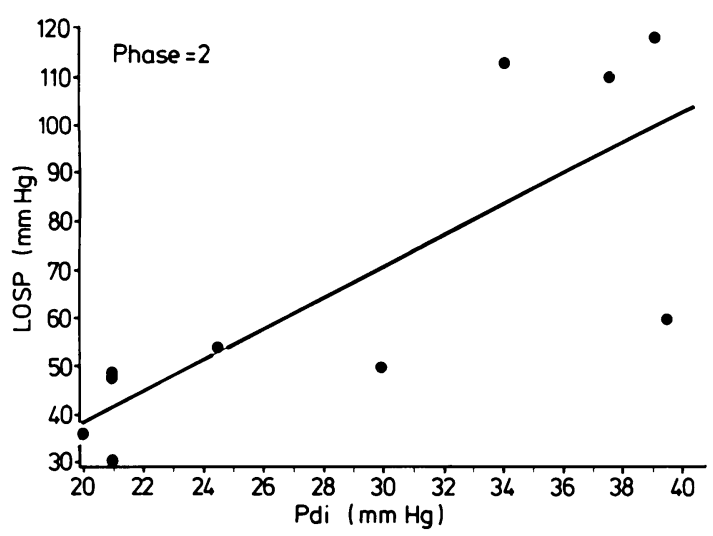

Fig. 3 Correlation between the transdiaphragmatic pressure ((Pdi), a measure of the force of diaphragmatic contraction), and lower oesophageal sphincter pressure (LOSP) in phase II. Each data point represent a mean of the five observations in each subject. The Pdiand LOSP significantly correlated in a linear fashion, $r=0 \cdot 77, p<0 \cdot 005$.

easily observed during endoscopy, but a similar demonstration during phỳsiologic pressure monitoring has been difficult and controversial. The difficulty stems from the fact that the LOS moves passively along with the diaphragm during inspiration and expiration, but the manometric catheter remains stationary. ${ }^{+9}$ This axial movement of the LOS in relationship to the manometric pressure measuring port results in a motion artifact in the LOS pressure recording. Winans ${ }^{*}$ and Dodds et $a l^{+4}$ found this motion artifact to be a major mechanism of the respiration induced pressure oscillations in the LOS. On the contrary, Boyle $e t \mathrm{al}^{3}$ found that the respiration induced pressure oscillations in the LOS still occurred even when axial motion of LOS relative to the manometric catheter was prevented by pinning the catheter in the LOS. Their study suggested that active diaphragmatic contraction contributes to the LOS pressure oscillations in the cat. Welch and Gray" concluded that both the axial movement of the LOS and diaphragmatic contraction contribute to the respiration induced pressure oscillations in the LOS.

A sleeve device was initially devised by Dent to counteract the problem of axial movements of the LOS relative to the catheter. As long as the LOS remains positioned over any portion of the $6 \mathrm{~cm}$ length of the sleeve it will measure the LOS pressure. Even though the response rate of the proximal part of the sleeve is very quick, however, the response rate at its distal end is rather slow. An in vitro study with the sleeve suggested to us that a continuous pressure had to be applied to the distal end for 17 seconds in order to measure a pressure of $150 \mathrm{mmHg}$. During diaphragmatic contraction, when the LOS would be expected to suddenly move from proximal end of the sleeve to its distal end, the actual LOS pressure and the effect of diaphragmatic squeeze can only be measured accurately if the diaphragmatic contraction is sustained. This phenomenon was actually observed in our studies. In phase II when the diaphragmatic contraction was sustained, the increase in LOS pressure was gradual until maximal pressure was attained, at which point the LOS pressure tracing showed a horizontal pattern. We think that the LOS pressure actually increases very rapidly as the diaphragm contracts but the slow rise of LOS pressure as observed on our recordings is most likely an artifact because of the slow response rate of the sleeve device at its distal end. The rapid fall of LOS pressure in phase III when there is relaxation of the diaphragm supports the hypothesis that the increase in LOS pressure in phase II was the result of diaphragmatic contraction.

The LOS pressure in phase III in our study was significantly lower than phase I although by only a small magnitude. This fall in LOS pressure could be explained by a change in the length tension relationship of the LOS smooth muscles" as a result of a constricting effect of the diaphragm. The other possibility is that the stretch receptors in the lung during phases II and III when the lungs were inflated may mediate reflex inhibition of the smooth muscle LOS. Boyle and colleagues have recently described such a reflex which is mediated through the vagus nerve. ${ }^{12}$

The magnitude of Pdi on inspiration to total lung capacity in our study is similar to that observed by other investigators. ${ }^{13}$ The transdiaphragmatic pressure (Pdi) is considered to be a measure of the force of diaphragmatic contraction..$^{1+15}$ We therefore expected that the Pdi should correlate with the increase in LOS pressure. We indeed found a strong correlation between LOS pressure and Pdi in phase II. This would be consistent with the concept that the diaphragmatic contraction acts as an 'external sphincter', enhancing the intrinsic LOS tone.

Acid reflux into the oesophagus was not observed in any of the phases, in spite of a pressure gradient in phase II that should promote the flow of contents from the stomach into the oesophagus. It should be noted that Pdi in fact represents the gastrooesophageal gradient and hence a driving force for the gastric contents to reflux into the oesophagus. In phase I, the Pdi or the gastro-oesophageal gradient was only about $4 \mathrm{mmHg}$. Therefore an actual LOS pressure of greater than $4 \mathrm{mmHg}$ should provide an effective antireflux barrier. The observation by Dent et al that the acid reflux does not occur unless an end expiratory LOS pressure falls to less than $5 \mathrm{mmHg}^{\text {1h }}$ would support the fact that under resting conditions 
an end expiratory LOS pressure of more than 5 $\mathrm{mmHg}$ is an effective antireflux barrier against a gastro-oesophageal pressure gradient of $4 \mathrm{mmHg}$. During deep inspiration, however, when the gastrooesophageal gradient is in the range of $30-40 \mathrm{mmHg}$, an augmentation of the LOS pressure is required to avoid the possibility of acid reflux. Our study suggests that it is during these periods that the LOS pressure increases and this increase is always greater than Pdi. The reflex increases in the LOS pressure could be an important component of the antireflux barrier.

Our data clearly suggest that the diaphragmatic contraction and relaxation results in a rise and fall of the LOS pressure. Whether these LOS pressure changes are the result of a sphincteric action of diaphragm or a reflex constriction of the smooth muscle of the LOS in response to diaphragmatic contraction is not totally clear. The latter may be possible because there are reflex pathways between the oesophagus and diaphragm ${ }^{17}$ and it is possible that a diaphragm to LOS reflex may exist as well.

The authors are indebted to Joyce HuYoung for her expertise in preparation of this manuscript. The data in this manuscript were presented at the American Motility Society Meeting 1986 in Houston and were published in abstract form in Gastroenterology 1986; 91: 1061 .

\section{References}

1 Goyal RK, Rattan S. Genesis of basal sphincter pressure: effect of tetrodotoxin on lower esophageal sphincter pressure in opossum in vivo. Gastroenterology 1976; 61: 62-7.

2 Clemeute CD. The diaphragm in Gray's Anatomy. 30th American Edition. Philadelphia: Lea \& Febiger, 1985: 479-80.

3 Boyle JT, Altschuler SM, Nixon TE, Tuchman DN, Pack AI, Cohen S. Role of the diaphragm in the genesis of lower esophageal sphincter pressure in the cat. Gastroenterology 1985; 88: 723-30.
4 Dodds WJ, Stewart ET, Hogan WJ, Steff JJ, Arndorfer RC. Effect of esophageal movement on intraluminal esophageal pressure recordings. Gastroenterology 1974; 67: 592-60).

5 Miranda JM, Lourenco RV. Influence of diaphragm activity on the measurement of total chest compliance. $J$ Appl Physiol 1968; 24: 741-6.

6 Milic-Emili J, Tyler JM. Relationship between the work output of respiratory muscles and end-tidal $\mathrm{CO}_{2}$ tension. J Appl Physiol 1963; 18: 497-5()4.

7 Dent J. A new technique for continuous sphincter pressure measurement. Gastroenterology 1976; 71: 263-7.

8 Winans CS. Alteration of lower esophageal sphincter characteristics with respiration and proximal esophageal balloon distension. Gastroenterology 1972; 62: 380-8.

9 Dodds WJ, Stewart ET, Hodges D, Zboralske FF. Movement of the feline esophagus associated with respiration and peristalsis. An evaluation using tantalum markers. J Clin Invest 1973; 52: 1-13.

10) Welch RW, Gray JE. Influence of respiration on recording of lower esophageal sphincter pressure in humans. Gastroenterology 1982; 83: 590-4.

11 Biancani P, Zabinski MP, Behar J. Pressure, tension, and force of closure of the human lower esophageal sphincter and esophagus. J Clin Invest 1975; 56: 476-83.

12 Boyle JT, Altschuler SM, Patterson BI, Pack AL, Cohen S. Reflex inhibition of the lower esophageal sphincter (LES) following stimulation of pulmonary vagal afferent receptors [Abstract]. Gastroenterology 1986; 90: 1353.

13 Detroyer A, Estenne M. Limitation of transdiaphragmatic pressure in detecting diaphragmatic weakness. Thorax 1981; 36: 169-74.

14 Bellemare F, Ritchie BB, Woods JJ. Contractile properties of the human diaphragm in vivo. J Appl Physiol 1986; 61: 1153-61.

15 Braun NM, Arora NS, Rochester DF. Force length relationship of the normal human diaphragm. $J$ Appl Physiol 1982; 53: 405-12.

16 Dent J, Dodds WJ, Friedman RH, et al. Mechanism of gastroesophageal reflux in recumbent asymptomatic human subjects. J Clin Invest 1980; 65: 256-67.

17 DeTroyer A, Rosso J. Reflux inhibition of the diaphragm by esophageal affarents. Neuroscience Letters 1982; 30: 43-6. 